\title{
A New Insight into the Coupler Curves of the RCCC Four-Bar Linkage
}

\author{
Federico Thomas ${ }^{1}$ and Alba Pérez-Gracia ${ }^{2}$ \\ ${ }^{1}$ Institut de Robòtica i Informàtica Industrial (CSIC-UPC), \\ Barcelona,08028 Spain; e-mail: fthomas@iri.upc.edu \\ ${ }^{2}$ Department of Mechanical Engineering, Idaho State University, Pocatello, \\ Idaho,83209 USA; e-mail: perealba@isu.edu
}

\begin{abstract}
Based on the condition for four points to lie on the unit sphere, derived using Distance Geometry, a new mathematical formulation for the coupler curves of the RCCC linkage is presented. The relevance of this formulation is not only its simplicity, but the elegant way in which we can obtain the derivative of any variable with respect to any other, and the simple way in which intervals of monotonicity can be detected. All these results are compactly expressed in terms of Gramians and, as a consequence, they have a direct geometric meaning contrarily to what happens with previous approaches based on kinematic loop equations.
\end{abstract}

Key words: RCCC linkage, coupler curves, Distance Geometry.

\section{Introduction}

The RCCC linkage is the most general spatial four-bar linkage with mobility one [1]. This linkage has been proved to be a good testbed in which to try and evaluate new ideas concerning the analysis of spatial linkages [2]. The spatial coupler curves of the RCCC linkage were studied in [3] using an analytic approach based on $3 \times 3$ orthogonal transformation matrices with dual number elements. This approach has become the standard formulation in most subsequent analysis of this linkage $[4,5]$.

In Fig. 1 appears a CCCC linkage from which an RCCC linkage can be derived by blocking the sliding motion of any of its cylindrical joints. In this linkage, the two parameters which describe the kinematics of the joint $\mathrm{i}$; namely, the angular displacement denoted as $\theta_{i}$ and the joint offset denoted as $d_{i}$ can be combined into the dual angle $\hat{\theta}_{i}=\theta_{i}+\varepsilon d_{i}$. The two parameters describing the geometry of link $i(i=1, \ldots, 4)$, namely, the twist angle denoted as $\theta_{i, i+1}$ and the link length denoted as $d_{i, i+1}$ can be combined into the dual angle $\hat{\theta}_{i, i+1}=\theta_{i, i+1}+\varepsilon d_{i, i+1}$ where $\varepsilon$ stands for the dual unit which is defined as $\varepsilon^{2}=0, \varepsilon \neq 0$. Observe that $\theta(d)$, with only a subindex, denotes the angular displacement (joint offset) of the joint defined by the subindex; 


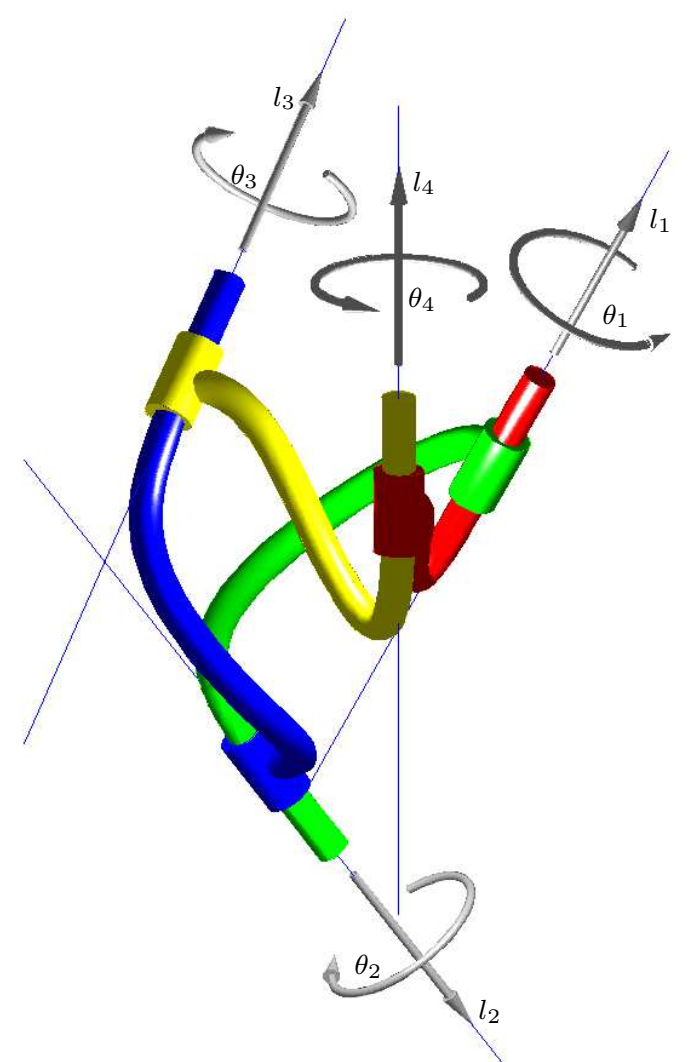

Fig. 1 Notation associated with the joints of a CCCC linkage.

and, with two subindices, denotes the twist angle (link length) between the two axes defined by the two subindices. This notation does not comply with the standard, but it simplifies the formulation given below and avoids possible confusions between standard and modified DH parameters. The axes $I_{1}$, $I_{2}$, and $I_{3}$, and $I_{4}$ can be transferred to the dual space by the four points $P_{1}, P_{2}, P_{3}$ and $P_{4}$, respectively, all lying on the dual unit sphere. Therefore, the position analysis of the CCCC linkage in the Euclidean space is equivalent to solving the position analysis of the corresponding single-dof spherical four-bar linkage in the dual unit sphere. To this end, it is possible to derive the loop equation of this spherical four-bar linkage as the product of $3 \times 3$ orthogonal transformation matrices with dual number elements. By developing this matrix product, a system of nine nonlinear equations in four unknowns $\left(\hat{\theta}_{i}, i=1, \ldots 4\right)$ is obtained $[6,7,3]$. Alternatively, we could also use dual quaternions in which case we would get four equations. Nevertheless, only three independent scalar equations are required to solve this position analysis because a four-bar linkage on the sphere has 1 dof. In this paper we depart from these standard formulations based on a loop equation by deriv- 
ing, using Distance Geometry on the sphere [8, 9], a single scalar equation in two unknowns. The relevance of this equation is not only its simplicity, but the elegant way in which we can obtain the devivatives of any of the variables with respect to any other, including the geometric interpretation of local extrema, in terms of Gramians.

The rest of this paper is organized as follows. Section 2 concisely describes the new formulation and its basic properties. Section 3 gives the main clues on how this new formulation can be applied to a particular example. Finally, Section 4 gives some conclusions and prospects for future research.

\section{Deriving a single scalar closure condition}

In spherical geometry, the shortest distance between two points, also known as the geodesic distance, is the length of an arc of a great circle containing both points. This great circle is the result of intersecting the plane passing through the origin and the two points with the sphere. Then, the distance between two points on the unit sphere is $d\left(P_{i}, P_{j}\right)=\cos ^{-1}\left\langle\mathbf{p}_{i}, \mathbf{p}_{j}\right\rangle$, the angle between the vectors from the origin to the points $P_{i}$ and $P_{j}$ which will be denoted by $\theta_{i j}$. Here $\left\langle\mathbf{p}_{i}, \mathbf{p}_{j}\right\rangle$ is the standard Euclidean inner product.

The triangle inequality between three points on a sphere holds provided that the distance between any two points is the lowest of the two arcs of great circle with them as endpoints. Then, if we assume that $0 \leq \theta_{i, j}<\pi$, the mapping between $\theta_{i, j}$ and $\cos \theta_{i, j}$ becomes one-to-one. Observe that a link with twist angle $\theta_{i, j}$ is kinematically equivalent to a link with twist angle $2 \pi-\theta_{i, j}$. Therefore, in what follows and as a matter of convenience, we will indistinctly use $\theta_{i j}$ or $\cos \theta_{i j}$ when referring to the distance between $P_{i}$ and $P_{j}$.

Given the location vectors, $\mathbf{p}_{1}, \ldots, \mathbf{p}_{n}$, of points $P_{1}, \ldots, P_{n}$, the Gram determinant, or Gramian, is the determinant defined as

$$
G(1, \ldots, n)=\left|\begin{array}{cccc}
\left\langle\mathbf{p}_{1}, \mathbf{p}_{1}\right\rangle & \left\langle\mathbf{p}_{1}, \mathbf{p}_{2}\right\rangle & \ldots & \left\langle\mathbf{p}_{1}, \mathbf{p}_{n}\right\rangle \\
\left\langle\mathbf{p}_{2}, \mathbf{p}_{1}\right\rangle & \left\langle\mathbf{p}_{2}, \mathbf{p}_{2}\right\rangle & \ldots & \left\langle\mathbf{p}_{2}, \mathbf{p}_{n}\right\rangle \\
\vdots & \vdots & \ddots & \vdots \\
\left\langle\mathbf{p}_{n}, \mathbf{p}_{1}\right\rangle & \left\langle\mathbf{p}_{n}, \mathbf{p}_{2}\right\rangle & \ldots & \left\langle\mathbf{p}_{n}, \mathbf{p}_{n}\right\rangle
\end{array}\right|
$$

which, in the particular case in which all points lie on the unit sphere, reduces to

$$
G(1, \ldots, n)=\left|\begin{array}{cccc}
1 & \cos \theta_{1,2} & \ldots & \cos \theta_{1, n} \\
\cos \theta_{1,2} & 1 & \ldots & \cos \theta_{2, n} \\
\vdots & \vdots & \ddots & \vdots \\
\cos \theta_{1, n} & \cos \theta_{2, n} & \ldots & 1
\end{array}\right|
$$


Gramians are zero if, and only if, the involved coordinate vectors are linearly dependent, and strictly positive otherwise [10, p. 251]. Negative Gramians only arise in those situations in which the given interpoint distances do not correspond to any configuration of real points. Since we limit our analysis to a sphere in three dimensions, a Gramian of four points necessarily vanishes. For the same reason, a Gramian of three points lying on a great circle also vanishes. Next, we analyze the four-point case in more detail.

If we have four points, say $P_{i}, P_{j}, P_{k}$ and $P_{l}$, then

$$
G(i, j, k, l)=\left|\begin{array}{cccc}
1 & \cos \theta_{i, j} & \cos \theta_{i, k} & \cos \theta_{i, l} \\
\cos \theta_{i, j} & 1 & \cos \theta_{j, k} & \cos \theta_{j, l} \\
\cos \theta_{i, k} & \cos \theta_{j, k} & 1 & \cos \theta_{k, l} \\
\cos \theta_{i, l} & \cos \theta_{j, k} & \cos \theta_{k, l} & 1
\end{array}\right|
$$

vanishes if, and only if, the four points lie on the three-dimensional unit sphere. Then, in what follows, $G(i, j, k, l)$ will be referred to as the closure condition for $P_{i}, P_{j}, P_{k}$ and $P_{l}$.

Using a computer algebra system, it can be verified that (see [11] for details)

$$
G(i, j) G(i, j, k, l)=\left(G(i, j) \cos \theta_{k, l}-B(i, j, k, l)\right)^{2}-G(i, j, k) G(i, j, l),
$$

where

$$
B(i, j, k, l)=\left|\begin{array}{ccc}
1 & \cos \theta_{i, j} & \cos \theta_{i, k} \\
\cos \theta_{i, j} & 1 & \cos \theta_{j, k} \\
\cos \theta_{i, l} & \cos \theta_{j, l} & 0
\end{array}\right|
$$

Now, since $G(i, j, k, l)=0$, we can easily conclude that

$$
\cos \theta_{k, l}=\frac{B(i, j, k, l)+\sigma_{k, l} \sqrt{G(i, j, l) G(i, j, k)}}{G(i, j)},
$$

where

$$
\sigma_{k, l}=\left\{\begin{aligned}
-1, & \text { if } P_{k} \text { and } P_{l} \text { lie on the two different hemispheres defined by } \\
& \text { the great circle containing } P_{i} \text { and } P_{j} \\
+1, & \text { otherwise }
\end{aligned}\right.
$$

If $G(i, j, k) G(i, j, l)=0$, there is only one solution for $\theta_{k, l}$. Clearly, this only happens when any of the two triangles $P_{i} P_{j} P_{k}$ and $P_{i} P_{j} P_{l}$ degenerate (i.e., when either $P_{k}$ or $P_{l}$ lies on the great circle defined by $P_{i}$ and $P_{j}$ ).

If we derive (4) with respect to $\cos \theta_{k, l}$, we conclude, using (6), that

$$
\frac{\partial G(i, j, k, l)}{\partial \cos \theta_{k, l}}=2 \sigma_{k, l} \sqrt{G(i, j, l) G(i, j, k)} .
$$



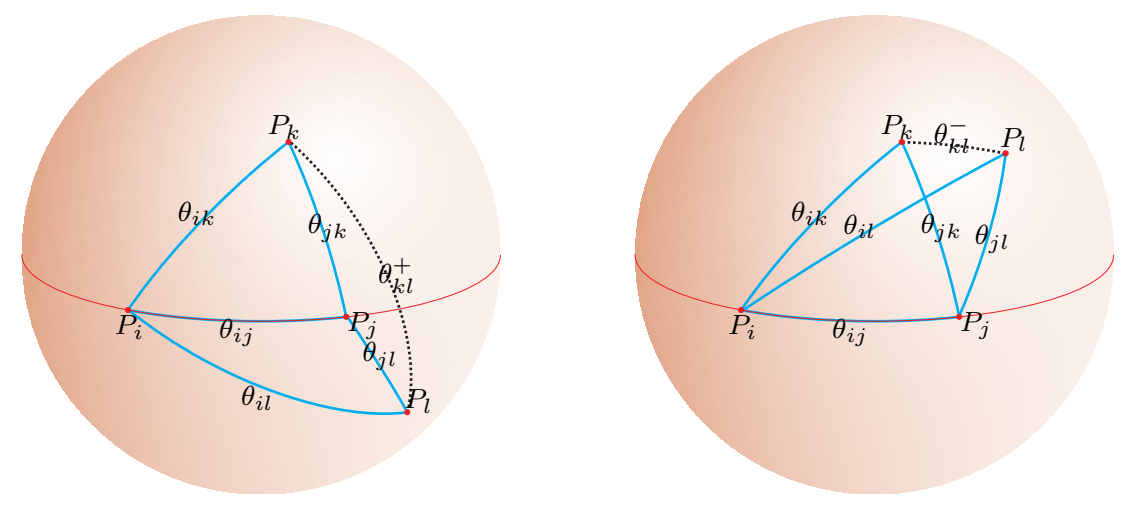

Fig. 2 Given four points on the unit sphere, $P_{i}, P_{j}, P_{k}$, and $P_{l}$, the distance between, say $P_{k}$ and $P_{l}$, is determined by all other interpoint distances. In general, two solutions are possible which corresponds to the cis (left) and trans configuration (right).

Similar expressions are obtained when deriving $G(i, j, k, l)$ with respect to the cosine of any other angle. Then, since $G(i, j, k, l)=0$, using the theorem of implicit differentiation, we have, for example, that

$$
\frac{\partial \cos \theta_{i, j}}{\partial \cos \theta_{k, l}}=-\frac{\partial G(i, j, k, l) / \partial \cos \theta_{k, l}}{\partial G(i, j, k, l) / \partial \cos \theta_{i, j}}=-\sigma_{i, j} \sigma_{k, l} \sqrt{\frac{G(i, j, l) G(i, j, k)}{G(i, k, l) G(j, k, l)}} .
$$

It follows from this expression that the variation of $\theta_{i, j}$ with respect to that of $\theta_{k, l}$ is monotone provided that no three points involved in the Gramians inside the squared root get aligned (they lie on a great circle).

The derivative in (8) is also very helpful to obtain the dual component of the closure condition $G(i, j, k, l)=0$ when extending it to dual angles. Let us denote this extension as $\widehat{G}(i, j, k, l)=0$. Then, $\widehat{G}(1,2,3,4)=0$, in the dual variables $\hat{\theta}_{i, j}=\theta_{i, j}-\varepsilon d_{i, j}$, for $1 \leq i \leq j \leq 4$, can be expressed using the chain rule as follows:

$$
\widehat{G}(1,2,3,4)=G(1,2,3,4)+\varepsilon \sum_{1 \leq i<j \leq 4} d_{i, j} \frac{\partial G(1,2,3,4)}{\partial \cos \theta_{i, j}} \frac{\partial \cos \theta_{i, j}}{\partial \theta_{i, j}} .
$$

This allows us to conclude, using (8), that the dual component of the closure condition can be expressed as

$$
\sum_{1 \leq i<j \leq 4} \sigma_{i, j} d_{i, j} \sin \theta_{i, j} \sqrt{G(\{1,2,3,4\} \backslash i) G(\{1,2,3,4\} \backslash j)}=0,
$$

an elegant expression for the dual component of $\widehat{G}(1,2,3,4)=0$ of great compactness when compared with the results obtained using loop equations. 


\section{Example}

Let us consider the CCCC linkage in Fig. 1 for which we want to obtain the coupler curve relating $\theta_{4}$ as a function of $\theta_{1}$. This problem corresponds to the second example in [3]. In this particular case,

$\hat{\theta}_{1,2}=30^{\circ}+\varepsilon 2 \mathrm{~cm}, \quad \hat{\theta}_{2,3}=120^{\circ}+\varepsilon 3 \mathrm{~cm}, \quad \hat{\theta}_{3,4}=105^{\circ}+\varepsilon 4 \mathrm{~cm}, \quad \hat{\theta}_{1,4}=45^{\circ}+\varepsilon 3.5 \mathrm{~cm}$, and where the input joint offset, $d_{1}$, is set to $6 \mathrm{~cm}$.

If we only consider the real part of the closure condition $\widehat{G}(1,2,3,4)=0$, i.e. $G(1,2,3,4)=0$, we can readily plot $\theta_{13}$ as a function of $\theta_{24}$ using (6). The result appears in Fig. 3. In this plot, the Cartesian region defined by $[-1,1] \times[-1,1]$ can be divided into subregions attending to the signs of the Gramians resulting from taking all subsets of three points. Only the part of the curve inside the region where all these Gramians are positive has physical meaning. The boundaries between all these regions correspond to configuration where three points get aligned. The curve itself can also be subdivided into monotonic segments attending to the signs of $\sigma_{1,3}$ and $\sigma_{2,4}$. The points separating these segments also correspond to points where three points get aligned. As a consequence, these points are necessarily located on the boundary of the feasibility region. In general, we can have up to four such segments, but in this particular example we just have three because this mechanism has a configuration in which $G(1,3,4)=G(1,2,3)=0$ which corresponds to the limiting position reported in [3]. At this position $\theta_{1}=\pi$ and the coupler link and the output link are collinear, i.e., $\theta_{3}=\pi$ (see [3] for more details). This mechanism has no configurations where $\sigma_{1,3}=+1$ and $\sigma_{2,4}=-1$, that is, configurations where $P_{1}$ and $P_{3}$ are located on the same hemisphere defined with respect to the great circle containing $P_{2}$ and $P_{4}$, and where $P_{2}$ and $P_{4}$ are located on different hemispheres defined with respect to the great circle containing $P_{1}$ and $P_{3}$.

Each point of the obtained curve gives a value for $\cos \theta_{24}$ and $\cos \theta_{13}$ from which we can obtain the values of $\cos \theta_{i}, i=1, \ldots, 4$, using the law of cosines for suplementary angles. In the particular case of $\cos \theta_{1}$ and $\cos \theta_{4}$ we have that:

$$
\begin{aligned}
\cos \theta_{1} & =\left(-\cos \theta_{2,4}+\cos \theta_{1,4} \cos \theta_{1,2}\right) /\left(\sin \theta_{1,4} \sin \theta_{1,2}\right) \\
& =\left(-\cos \theta_{2,4}+0.61237\right) / 0.35355 \\
\cos \theta_{4} & =\left(-\cos \theta_{1,3}+\cos \theta_{3,4} \cos \theta_{1,4}\right) /\left(\sin \theta_{3,4} \sin \theta_{1,4}\right) \\
& =\left(-\cos \theta_{1,3}-0.18301\right) / 0.68301
\end{aligned}
$$

Now, we can obtain the plot expressing $\cos \theta_{4}$ as a function of $\cos \theta_{1}$ that appears in Fig. 4(left). Since (12) and (13) are affine relations, the monotonicity of the three segments is preserved. 


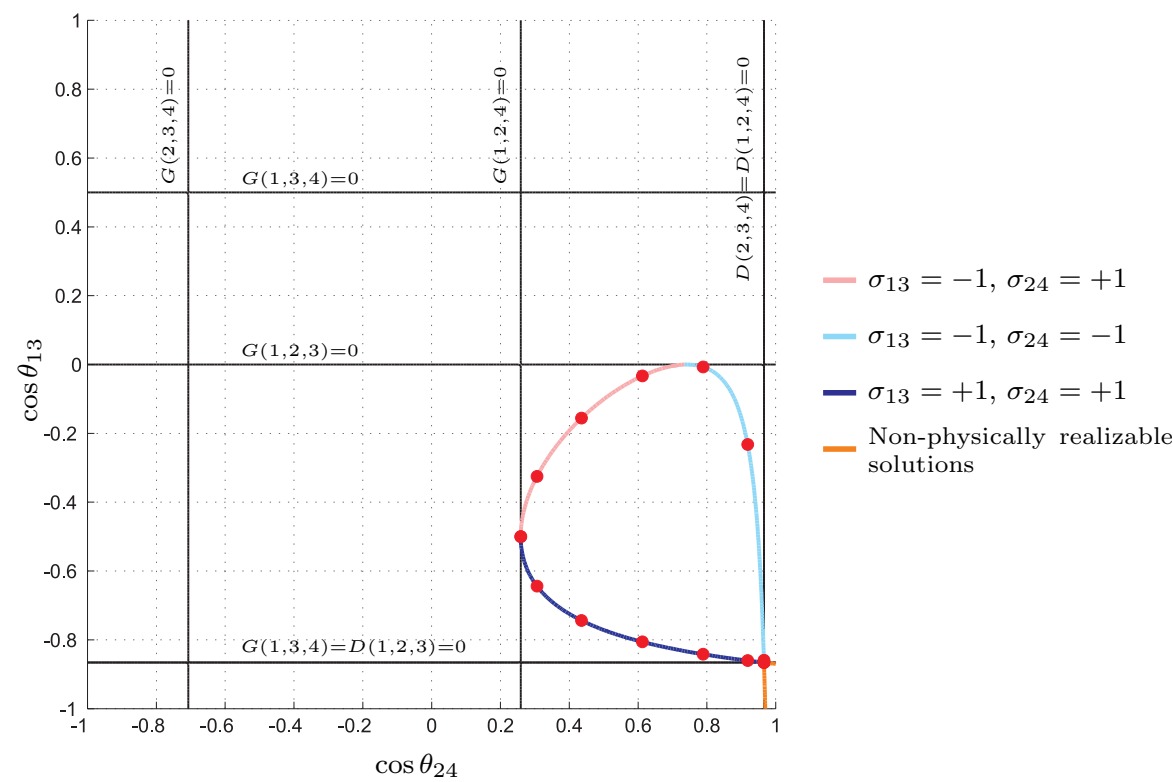

Fig. 3 Plot of $\cos \theta_{1,3}$ as a function of $\cos \theta_{2,4}$ (it can also be seen as the root locus of $G(1,2,3,4)=0)$. The dots in red correspond to the linkage configurations reported in [3].
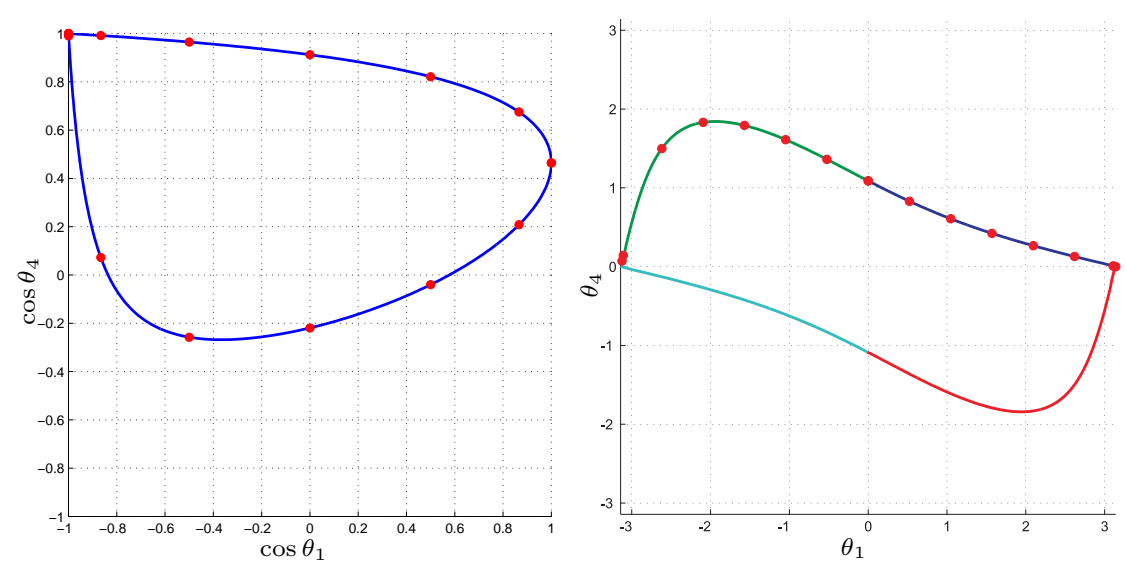

Fig. 4 Left: Curve relating $\cos \theta_{4}$ and $\cos \theta_{1}$ obtained from the curve in Fig. 3 using (13) and (12). Right: Coupler curve relating $\theta_{1}$ and $\theta_{4}$. Again, the dots in red correspond to the linkage configurations reported in [3].

Finally, the coupler curve relating $\theta_{4}$ and $\theta_{1}$ can be obtained by computing the \pm arccos of both coordinates of each point of the curve in Fig. 4(left). This is a 1-to-4 mapping. Two of these images are not solutions of the problem because they do not satisfy the original kinematic constraints, i.e. the signs of $\theta_{1,2}, \theta_{2,3}, \theta_{3,4}$, and $\theta_{1,4}$. As with many problems solved in a distance space, 
when mapping the obtained solutions to an Euclidean space, they should be checked to satisfy some consistency constraints [12].

\section{Conclusion}

The position analysis of a RCCC linkage in the Euclidean space is equivalent to solving the position analysis of a spherical four-bar linkage in the dual unit sphere. If we try to solve this problem using Distance Geometry directly in the Euclidean space using Cayley-Menger determinants, the formulation becomes quite involved. In this paper, we have shown how the problem can be solved using Distance Geometry in the dual unit sphere. This result opens the possibility of using Cayley-Menger determinants with dual arguments to compactly formulate other geometric problems. This is a point that certainly deserves further attention.

Acknowledgements This work has been partially supported by the Spanish Ministry of Economy and Competitiveness under project DPI2014-57220-C2-2-P, and by the National Science Foundation under Grant No. 1208385. The content of this paper is solely the authors' responsibility.

\section{References}

1. A.T. Yang and F. Freudenstein, "Application of dual-number quaternion algebra to the analysis of spatial mechanisms," ASME J. of Applied Mechanics, Series E, vol. 2, pp. 300-308, 1964.

2. R.I. Jamalov, F.L. Litvin, and B. Roth, "Analysis and design of RCCC linkages," Mechanism and Machine Theory, vol. 19, no. 4-5, pp. 397-407.

3. S.D. Marble and G.N. Pennock, "Algebraic-geometric properties of the coupler curve of the RCCC spatial four-bar mechanism," Mech. Mach. Theory, vol. 35, no. 5, pp. 675-693, 2000.

4. S. Bai and J. Angeles, "A unified input-output analysis of four-bar linkages," Mechanism and Machine Theory, Vol. 43, No. 2, pp. 240-251, 2008.

5. G. Figliolini, P. Rea and J. Angeles, "The Synthesis of the Axodes of RCCC Linkages," ASME J. Mechanisms Robotics, vol. 8, no. 2, paper 021011 (9 pages), 2015.

6. F. Thomas, "On the n-bar mechanism, or how to find global solutions to redundant single loop spatial kinematic chains," IEEE Int. Conf. on Robotics and Automation, Vol. I, pp. 403-408, 1992.

7. I.S. Fischer Dual-Numer Methods in Kinematics, Statics and Dynamics, CRC Press, New York, 1998.

8. D. Audet, "Déterminants sphérique et hyperbolique de Cayley-Menger," Bulletin de l'Association Mathématique du Québec, Vol. LI, no. 2, pp. 45-52, 2011.

9. H. Maehara, "On spherical four-point-spaces," Journal of Geometry, Vol. 107, no. 1, pp. 1-8, 2016.

10. F. R. Gantmakher, The Theory of Matrices, Volumen 1, Chelsea Pub Co, 1984.

11. J.M. Porta and F. Thomas, "Distance-bound smoothing on the sphere," IEEE Int. Conf. on Robotics and Automation, Singapore, 2017.

12. N. Rojas, Distance-Based Formulations for the Position Analysis of Kinematic Chains, Technical University of Catalonia, Spain, 2012. 